\title{
ON THE HIRSCH-PLOTKIN RADICAL OF A FACTORIZED GROUP
}

\author{
by SILVANA FRANCIOSI and FRANCESCO DE GIOVANNI
}

(Received 27 November, 1990)

1. Introduction. Let the group $G=A B$ be the product of two subgroups $A$ and $B$. A normal subgroup $K$ of $G$ is said to be factorized if $K=(A \cap K)(B \cap K)$ and $A \cap B \leq K$, and this is well-known to be equivalent to the fact that $K=A K \cap B K$ (see [1]). Easy examples show that normal subgroups of a product of two groups need not, in general, be factorized. Therefore the determination of certain special factorized subgroups is of relevant interest in the investigation concerning the structure of a factorized group. In this direction E. Pennington [5] proved that the Fitting subgroup of a finite product of two nilpotent groups is factorized. This result was extended to infinite groups by B. Amberg and the authors, who proved in [2] that if the soluble group $G=A B$ with finite abelian section rank is the product of two locally nilpotent subgroups $A$ and $B$, then the Hirsch-Plotkin radical (i.e. the maximum locally nilpotent normal subgroup) of $G$ is factorized. If $G$ is a soluble $\mathscr{S}_{1}$-group and the factors $A$ and $B$ are nilpotent, it was shown in [3] that also the Fitting subgroup of $G$ is factorized. However, Pennington's theorem becomes false for finite soluble groups which are the product of two arbitrary subgroups. For instance, the symmetric group of degree 4 is the product of a subgroup isomorphic with the symmetric group of degree 3 and a cyclic subgroup of order 4, but its Fitting subgroup is not factorized.

The aim of this paper is to prove that even in the case of a group factorized by two arbitrary subgroups the Hirsch-Plotkin radical and the Fitting subgroup have some factorization properties.

Theorem A. Let the soluble-by-finite group $G=A B$ with finite abelian section rank be the product of two subgroups $A$ and $B$, and let $H$ be the Hirsch-Plotkin radical of $G$. Then $H=A_{0} H \cap B_{0} H$, where $A_{0}$ and $B_{0}$ are the Hirsch-Plotkin radicals of $A$ and $B$, respectively.

Here the requirement that $G$ has finite abelian section rank cannot be removed, as Ya. P. Sysak [10] gave an example of a triply factorized group $G=A B=A K=B K$, where $A, B$ and $K$ are torsion-free abelian subgroups and $K$ is normal in $G$, but $G$ is not locally nilpotent.

In the hypotheses of Theorem $A$, if the subgroups $A$ and $B$ are locally nilpotent, one has in particular that the Hirsch-Plotkin radical of $G$ is factorized. Similarly, the factorization of the Fitting subgroup of a soluble $\mathscr{S}_{1}$-group factorized by two nilpotent subgroups is a consequence of the following result.

THEOREM B. Let the soluble-by-finite $\mathscr{S}_{1}$-group $G=A B$ be the product of two subgroups $A$ and $B$, and let $F$ be the Fitting subgroup of $G$. Then $F=A_{0} F \cap B_{0} F$, where $A_{0}$ and $B_{0}$ are the Fitting subgroups of $A$ and $B$, respectively.

Most of our notation is standard and can for instance be found in [6]. In particular:

If $G$ is a group, $\bar{Z}(G)$ is the hypercentre of $G$.

If $G$ is a group, $\pi(G)$ is the set of prime divisors of the orders of elements of $G$. 
A group $G$ has finite abelian section rank if it has no infinite elementary abelian $p$-sections for every prime $p$.

A group $G$ is an $\mathscr{S}_{1}$-group is it has finite abelian section rank and the set of primes $\pi(G)$ is finite.

If $Q$ is a group and $M$ is a $Q$-module, $H_{n}(Q, M)$ and $H^{n}(Q, M)$ are the $n$-th homology group and the $n$-th cohomology group of $Q$ with coefficients in $M$, respectively.

If $N$ is a normal subgroup of a factorized group $G=A B$, the factorizer of $N$ in $G$ is the subgroup $X(N)=A N \cap B N$.

2. Proof of the Theorems. Our first lemma shows that Theorems A and B hold in the finite case.

LemMa 1. Let the finite group $G=A B$ be the product of two subgroups $A$ and $B$, and let $F$ be the Fitting subgroup of $G$. Then $F=A_{0} F \cap B_{0} F$, where $A_{0}$ and $B_{0}$ are the Fitting subgroups of $A$ and $B$, respectively.

Proof. Assume that the lemma is false, and let $G=A B$ be a counterexample of minimal order. If $N_{1}$ and $N_{2}$ are distinct minimal normal subgroups of $G$, and $F_{i} / N_{i}$ is the Fitting subgrup of $G / N_{i}(i=1,2)$, it follows that $A_{0} F_{i} \cap B_{0} F_{i}=F_{i}$, since the result holds for the factor group $G / N_{i}$. Then

$$
A_{0} F \cap B_{0} F \leq F_{1} \cap F_{2}=F,
$$

and $F=A_{0} F \cap B_{0} F$. This contradiction shows that $G$ has a unique minimal normal subgroup $N$, and hence $F$ is a $p$-group for some prime $p$. Put $F_{0}=A_{0} F \cap B_{0} F$. Since $F \leq F_{0} \leq A_{0} F$, the subgroup $F_{0}$ is subnormal in $A F$, and similarly it is subnormal in $B F$. Then it follows from Satz 1 of [11] that $F_{0}$ is subnormal also in the factorized group $G=(A F)(B F)$. Therefore $F_{0}$ is not nilpotent, and there exists a prime $q \neq p$ dividing the order of $F_{0}$. The Sylow $q$-subgroup $Q_{1}$ of $A_{0}$ is clearly also a Sylow $q$-subgroup of $A_{0} F$, and hence $Q=Q_{1} \cap F_{0}$ is a Sylow $q$-subgroup of $F_{0}$. Moreover $Q$ lies in $A_{0}$, and so is subnormal in $A$. Let $Q_{2}$ be the Sylow $q$-subgroup of $B_{0}$. Then $Q_{2}$ is a Sylow $q$-subgroup of $B_{0} F$, and thus there exists $x \in G$ such that

$$
Q \leq Q_{2}^{x} \leq B_{0}^{x}
$$

As $B_{0}^{x}$ is the Fitting subgroup of $B^{x}$, we obtain that $Q$ is subnormal in $B^{x}$, and Satz 1 of [11] yields that $Q$ is subnormal in $G=A B^{x}$. Since $F$ is a $p$-group, it follows that $Q=1$, and this contradiction proves the lemma.

Lemma 2. Let the group $G=A B=A K=B K$ be the product of two subgroups $A$ and $B$ and a radicable abelian normal p-subgroup $K$ satisfying the minimal condition. If $A_{0}$ and $B_{0}$ are nilpotent normal subgroups of $A$ and $B$, respectively, then the subgroup $A_{0} K \cap B_{0} K$ is nilpotent.

Proof. Assume that the lemma is false, and choose a counterexample

$$
G=A B=A K=B K
$$

such that $K$ has minimal Prüfer rank. Clearly the subgroups $A_{0} K$ and $B_{0} K$ are normal in $G$, and hence also $K_{0}=A_{0} K \cap B_{0} K$ is a normal subgroup of $G$. Moreover $K_{0} / K \leq$ 
$A_{0} K / K$ is obviously nilpotent. Suppose that $K_{0}$ is finite-by-nilpotent. Then there exists a positive integer $r$ such that the index $\left|K_{0}: Z_{r}\left(K_{0}\right)\right|$ is finite (see [6] Part 1, Theorem 4.25), so that $K \leq Z_{r}\left(K_{0}\right)$ and $K_{0}$ is nilpotent. This contradiction shows that $K_{0}$ is not finite-by-nilpotent. Let $L$ be an infinite $G$-invariant subgroup of $K$ with minimal Prüfer rank. Then $L$ is radicable and all its proper $G$-invariant subgroups are finite. By the minimality of the rank of $K$ the result holds for the factor group $G / L$, and hence $K_{0} / L$ is nilpotent. It follows that $\left[L, K_{0}\right] \neq 1$, and so $\left[L, K_{0}\right]=L$, since $\left[L, K_{0}\right]$ is radicable and $L$ has no infinite proper $G$-invariant subgroups. This means that $H_{0}\left(K_{0} / L, L\right)=0$, and Theorem $\mathrm{C}$ of [8] yields that $H^{2}(G / L, L)$ has finite exponent. Therefore there exists a subgroup $J$ of $G$ such that $G=L J$ and $L \cap J$ is finite. As $L \cap J$ is normal in $G$ and $K_{0}$ is not finite-by-nilpotent, also the factor group $G /(L \cap J)$ is a counterexample, and hence we may suppose that $L \cap J=1$. Thus $K=L \times(J \cap K)$ and $J \cap K \simeq K / L$ is a radicable normal subgroup of $G$. If $J \cap K \neq 1$, the result holds for the factor group $G /(J \cap K)$, and so $K_{0} /(J \cap K)$ is nilpotent. It follows that $K_{0}$ is nilpotent, and this contradiction proves that $J \cap K=1$. Therefore $K=L$, and $K$ has no infinite proper $G$-invariant subgroups. Assume that $A \cap K$ is infinite. As $A \cap K$ is normal in $G=A K$, we obtain that $A \cap K=K$ and $K \leq A$. Then $A_{0} K$ is nilpotent, so that also $K_{0}$ is nilpotent. This contradiction shows that $A \cap K$ is finite, and similarly $B \cap K$ is finite. Thus the normal subgroup $N=(A \cap K)(B \cap K)$ of $G$ is also finite, and as above the factor group $G / N$ is a counterexample. Hence we may suppose that $A \cap K=B \cap K=1$. If $A_{1}$ and $B_{1}$ are the Fitting subgroups of $A$ and $B$, respectively, it follows that $A_{1} K=B_{1} K$ is a normal subgroup of $G$ containing $K_{0}$. Since $H_{0}\left(K_{0} / K, K\right)=0$, application of Theorem C of [8] yields that $H^{1}\left(A_{1} K / K, K\right)$ has finite exponent. But $K$ is a radicable abelian $p$-group of finite rank, and hence there exists a finite characteristic subgroup $E$ of $K$ such that the complements of $K / E$ in $A_{1} K / E$ are conjugate (see [7]). The factor group $G / E$ is also a counterexample, so that we may suppose that the complements of $K$ in $A_{1} K$ are conjugate. As $A_{1}$ and $B_{1}$ are both complements of $K$ in $A_{1} K$, there exists $x \in G$ such that $A_{1}^{x}=B_{1}$. Write $x=a b$, where $a \in A$ and $b \in B$. Then

$$
A_{1}=A_{1}^{a}=B_{1}^{b^{-1}}=B_{1},
$$

so that $A_{1}=B_{1}$ is normal in $G$, and $A_{1} K$ is nilpotent. This last contradiction completes the proof of the lemma.

Lemma 3. Let $G$ be a group, and let $K$ be a periodic abelian normal subgroup of infinite exponent of $G$ whose proper $G$-invariant subgroups are finite. Then $K$ is contained in the centre of the Fitting subgroup of $G$. In particular, if $C_{G}(K)=K$, then $K$ is the Fitting subgroup of $G$.

Proof. Let $N$ be a nilpotent normal subgroup of $G$. Then $K N$ is also nilpotent, and hence $K \cap Z(K N)$ is infinite, since $K$ has infinite exponent (see for instance [6], Theorem 2.23). But $K \cap Z(K N)$ is normal in $G$, and $K$ has no infinite proper $G$-invariant subgroups, so that $K \cap Z(K N)=K$. Therefore $K \leq Z(K N)$ and $N \leq C_{G}(K)$. This proves that $K$ lies in the centre of the Fitting subgroup of $G$.

Proof of Theorem A. Assume that the result is false, and among all the counterexamples for which the soluble radical $S$ of $G$ has minimal index choose one $G=A B$ such that $S$ has minimal derived length. As the theorem is true for finite groups 
by Lemma 1 , the group $G$ is infinite, and hence its soluble radical is not trivial. It follows that $G$ contains an abelian normal subgroup $K$ such that the theorem holds for the factor group $G / K$. Write $M=A_{0} H \cap B_{0} H$. Then $M / K$ lies in the Hirsch-Plotkin radical of $G / K$, and hence $M$ is ascendant in $G$, as the Hirsch-Plotkin radical of $G / K$ is hypercentral. Since $H<M$, this proves that $M$ is not locally nilpotent. The factorizer $X(H)$ of $H$ in $G=A B$ has a triple factorization

$$
X(H)=\bar{A} \bar{B}=\bar{A} H=\bar{B} H,
$$

where $\bar{A}=A \cap B H$ and $\bar{B}=B \cap A H$. If $\bar{A}_{0}=A_{0} \cap \bar{A}=A_{0} \cap B H$ and $\bar{B}_{0}=B_{0} \cap \bar{B}=$ $B_{0} \cap A H$, then $\bar{A}_{0}$ and $\bar{B}_{0}$ are contained in the Hirsch-Plotkin radicals of $\bar{A}$ and $\bar{B}$, respectively. Moreover

$$
\bar{A}_{0} H \cap \bar{B}_{0} H=\left(A_{0} \cap B H\right) H \cap\left(B_{0} \cap A H\right) H=A_{0} H \cap B_{0} H=M,
$$

so that $\bar{A}_{0} H \cap \bar{B}_{0} H$ is not locally nilpotent. Therefore $X(H)=\bar{A} \bar{B}$ is also a minimal counterexample, and without loss of generality we may suppose that $G$ has a triple factorization

$$
G=A B=A H=B H .
$$

Then the subgroups $A_{0} H$ and $B_{0} H$ are normal in $G$, and hence also $M$ is a normal subgroup of $G$. The structure of soluble groups with finite abelian section rank (see [6]) allows us to investigate only the following possible choices for $K$.

Case 1: $K$ is finite. By induction on the order of $K$ be may suppose that $K$ is a minimal normal subgroup of $G$. As $M$ is not locally nilpotent, we have that $[K, M] \neq 1$ and hence $[K, M]=K$. Then $H_{0}(M / K, K)=0$, and it follows from Theorem 3.4 of $[9]$ that $H^{2}(G / K, K)=0$. Therefore there exists a subgroup $J$ of $G$ such that $G=K J$ and $K \cap J=1$. The centralizer $C_{J}(K)$ is normal in $G$, and Lemma 1 shows that the theorem holds for the finite factor group $G / C_{J}(K)$. In particular $M C_{J}(K) / C_{J}(K)$ is locally nilpotent, and so $M$ is locally nilpotent since $K \cap C_{J}(K)=1$. This contradiction proves that the subgroup $K$ cannot be finite.

Case 2: $K$ is periodic and residually finite. Each primary component $K_{p}$ of $K$ is finite, and so by Case 1 the group $M / K_{p}$, is locally nilpotent for every prime $p$. As the groups $K_{p}$ and $K / K_{p}$, are $G$-isomorphic, it follows that $K_{p}$ is hypercentrally embedded in $M$. Then $K$ is hypercentrally embedded in $M$, and $M$ is locally nilpotent, a contradiction.

Case 3: $K$ is a radicable $p$-group ( $p$ prime). By induction on the rank of $K$ we may suppose that every proper $G$-invariant subgroup of $K$ is finite. In particular, as $K$ is not hypercentrally embedded in $M$, the intersection $\bar{Z}(M) \cap K$ is finite. It follows from Case 1 that also the factor group $G /(\bar{Z}(M) \cap K)$ is a counterexample, and hence it can be assumed that $Z(M) \cap K=1$. Thus $H^{0}(M / K, K)=0$. Moreover, $G / C_{G}(K)$ is isomorphic with an irreducible linear group by Lemma 5 of [4], and hence it is abelian-by-finite (see [6] Part 1, Theorem 3.21). Then $M / C_{M}(K)$ is FC-hypercentrally embedded in $G$, and Theorem 3.5 of [9] yields that $H^{2}(G / K, K)=0$. Therefore there exists a subgroup $J$ of $G$ such that $G=K J$ and $K \cap J=1$. The centralizer $C_{J}(K)$ is normal in $G$, and $M C_{J}(K) / C_{J}(K)$ is not locally nilpotent. Put $\tilde{G}=G / C_{J}(K)$. As $K$ and $\tilde{K}$ are isomorphic $M$-modules, we obtain that $Z(\tilde{M}) \cap \bar{K}=1$. Moreover $C_{\tilde{G}}(\tilde{K})=\tilde{K}$, and replacing $G$ by $\tilde{G}$ we may suppose that $C_{G}(K)=K$ and $Z(M) \cap K=1$. In particular $K$ is the Fitting 
subgroup of $G$ by Lemma 3, and the factor group $G / K$ is abelian-by-finite. Let $L / K$ be an abelian normal subgroup of $G / K$ such that $G / L$ is finite. For each positive integer $n$, the $n$-th term $Z_{n}(H)$ of the upper central series of $H$ is a nilpotent normal subgroup of $G$, so that $Z_{n}(H) \leq K$. On the other hand, $K$ lies in $Z_{\omega}(H)$, since $H$ is hypercentral, and so $K=Z_{\omega}(H)$. Assume that $Z\left(A_{0}\right) \cap K$ contains a non-trivial element $a$, and let $m$ be the least positive integer such that $a \in Z_{m}(H)$. Then $Z_{m-1}(H)$ is properly contained in $K$, and hence is finite. Write $\bar{G}=G / Z_{m-1}(H)$. Then $\bar{a}$ centralizes $\bar{A}_{0}$ and $\bar{H}$, so $\bar{a} \in Z(\bar{M}) \cap \bar{K}$ and $Z(\bar{M}) \cap \bar{K} \neq 1$. As $Z_{m-1}(H)$ is finite and $Z(M) \cap K=1$, this contradicts Lemma 2.3 of [2]. Therefore $Z\left(A_{0}\right) \cap K=1$ and hence also $A_{0} \cap K=1$. But $A \cap K$ is contained in $A_{0}$, so that $A \cap K=1$. The same argument shows that $B \cap K=1$. Then the subgroups $A$ and $B$ are abelian-by-finite, and in particular the indices $\left|A: A_{0}\right|$ and $\left|B: B_{0}\right|$ are finite. The factorizer $X=X(K)$ of $K$ in $G=A B$ has a triple factorization

$$
X=A^{*} B^{*}=A^{*} K=B^{*} K,
$$

where $A^{*}=A \cap B K$ and $B^{*}=B \cap A K$. It follows from Lemma 2 that $A_{0} K \cap B_{0} K=$ $\left(A_{0} \cap B K\right) K \cap\left(B_{0} \cap A K\right) K$ is nilpotent-by-finite and hence $X$ is also. Thus the Fitting subgroup $Y$ of $X$ is nilpotent and $X / Y$ is finite. As $K \leq Y \cap L \leq L$, we have that $Y \cap L$ is a nilpotent normal subgroup of $L$. Clearly $K$ is the Fitting subgroup of $L$, so that $Y \cap L=K$, and $K$ has finite index in $X$. But $A^{*} \cap K=B^{*} \cap K=1$, so that $A^{*}$ and $B^{*}$ are finite, and $X=A^{*} B^{*}$ is also finite. This contradiction completes the proof of this case.

Case 4: $K$ is a periodic radicable group. Each primary component $K_{p}$ of $K$ is radicable, so that Case 3 shows that $M / K_{p}$, is locally nilpotent for every prime $p$. Then $K / K_{p}$ is hypercentrally embedded in $M$, and hence $K_{p}$ lies in the hypercentre of $M$. It follows that $K$ is hypercentrally embedded in $M$, and $M$ is locally nilpotent.

Case 5: $K$ is torsion-free. Let $T$ be the maximum periodic normal subgroup of $G$. As $K \cap T=1$, we have that $M T / T$ is not locally nilpotent, and hence the factor group $G / T$ is also a counterexample. Thus we may suppose that $G$ has no non-trivial periodic normal subgroups, so that in particular the set of primes $\pi(G)$ is finite (see [6] Part 2, Lemma 9.34). It follows that $G$ is nilpotent-by-polycyclic-by-finite (see [6] Part 2, Theorem 10.33). If $F$ is the Fitting subgroup of $G$, then $K \cap Z(F) \neq 1$. Consider a non-trivial element $x$ of $K \cap Z(F)$, and let $N$ be the normal closure of $x$ in $G$. Thus $N$ is a cyclic module over the polycyclic-by-finite group $G / F$, and hence it contains a free abelian subgroup $E$ such that $N / E$ is a $\pi$-group, where $\pi$ is a finite set of primes (see [6] Part 2, Corollary 1 to Lemma 9.53). Clearly

$$
\left(\bigcap_{p \notin \pi} N^{p}\right) \cap E=\bigcap_{p \notin \pi}\left(N^{p} \cap E\right)=\bigcap_{p \notin \pi} E^{p}=1,
$$

so that $\bigcap_{p \notin \pi} N^{p}$ is periodic, and $\bigcap_{p \notin \pi} N^{p}=1$ since $N \leq K$ is torsion-free. Let $p$ be any prime which does not belong to $\pi$. As $N^{p} \neq 1$, by induction on the torsion-free rank of $G$ we may suppose that the theorem holds for $G / N^{p}$. Therefore $M / N^{p}$ is locally nilpotent. Let $r$ be the Prüfer rank of $N$. Then $\left|N / N^{p}\right|=p^{r}$, so that $N / N^{p}$ lies in the $r$-th term of the upper central series of $M / N^{p}$. It follows that

$$
[N, \underbrace{M, \ldots, M}_{r}] \leq \bigcap_{p \notin \pi} N^{p}=1,
$$


and so $N \leq Z_{r}(M)$. Thus $M$ is locally nilpotent, and this last contradiction completes the proof of Theorem A.

Proof of Theorem $B$. Assume that the result is false, and choose a counterexample $G=A B$ such that the radicable part $R$ of the maximum periodic normal subgroup of $G$ has minimal total rank. Put $F_{0}=A_{0} F \cap B_{0} F$. Then Theorem A proves that $F_{0}$ lies in the Hirsch-Plotkin radical of $G$, and hence is locally nilpotent. The periodic subgroups of the factor group $G / R$ are finite, so that the Hirsch-Plotkin radical and the Fitting subgroup of $G / R$ coincide (see [6] Part 2, p. 35), and it follows again from Theorem A that $F_{0} / R$ is contained in the Fitting subgroup of $G / R$. As the Fitting subgroup of an $\mathscr{S}_{1}$-group is nilpotent, we obtain that $F_{0}$ is subnormal in $G$ and $F_{0} / R$ is nilpotent. Also, in an $\mathscr{S}_{1}$-group each nilpotent subnormal subgroup lies in the Fitting subgroup, so $F_{0}$ is not nilpotent and $R \neq 1$. Since $F_{0}$ is locally nilpotent, we have also that $F_{0}$ is not finite-by-nilpotent. Let $S$ be an infinite $G$-invariant subgroup of $R$ with minimal total rank, Then $S$ is a radicable abelian $p$-group for some prime $p$, and all its proper $G$-invariant subgroups are finite. Thus $G / C_{G}(S)$ is isomorphic with an irreducible linear group by Lemma 5 of [4], and hence it is abelian-by-finite. Moreover $G / S$ is an $\mathscr{S}_{1}$-group, so that its Fitting subgroup $F_{1} / S$ is nilpotent and $F_{0} \leq F_{1}$ by the minimal choice of $G$. Therefore $\left[S, F_{1}\right] \neq 1$, and hence $\left[S, F_{1}\right]=S$. Thus $H_{0}\left(F_{1} / S, S\right)=0$, and Theorem $\mathrm{C}$ of $[8]$ yields that $H^{2}(G / S, S)$ has finite exponent. Then there exists a subgroup $J$ of $G$ such that $G=S J$ and $S \cap J$ is finite. The subgroup $S \cap J$ is normal in $G$, and the factor group $G /(S \cap J)$ is also a counterexample, since $F_{0}$ is not finite-by-nilpotent. Therefore we may suppose that $S \cap J=1$, so that $R=S \times(J \cap R)$, where $J \cap R$ is a radicable normal subgroup of $G$. Clearly $F_{0}(J \cap R) /(J \cap R)$ is not nilpotent, so $J \cap R=1$ and $R$ has no infinite proper $G$-invariant subgroups. The centralizer $C_{J}(R)$ is normal in $G$, and the periodic subgroups of $J / C_{J}(R)$ are finite (see [6] Part 1, Corollary to Lemma 3.28), so that $G / C_{J}(R)$ is an $\mathscr{S}_{1}$-group. As $C_{J}(R) \cap R=1$, the group $F_{0} C_{J}(R) / C_{J}(R)$ is not nilpotent, and the theroem is false for the group $G / C_{J}(R)$. Clearly $R$ is $G$-isomorphic with the radicable part of the maximum periodic normal subgroup of $G / C_{J}(R)$, so that $G / C_{J}(R)$ is also a minimal counterexample. Moreover

$$
C_{J / C_{(}(R)}\left(R C_{J}(R) / C_{J}(R)\right)=1,
$$

and hence we may suppose that $C_{J}(R)=1$ and $C_{G}(R)=R$. Thus it follows from Lemma 3 that $R$ is the Fitting subgroup of $G$. The factorizer $X=X(R)$ of $R$ in $G$ has the triple factorization

$$
X=A^{*} B^{*}=A^{*} R=B^{*} R,
$$

where $A^{*}=A \cap B R$ and $B^{*}=B \cap A R$. Write $A_{0}^{*}=A_{0} \cap B R$ and $B_{0}^{*}=B_{0} \cap A R$. Then $A_{0}^{*}$ and $B_{0}^{*}$ are nilpotent normal subgroups of $A^{*}$ and $B^{*}$, respectively, and Lemma 2 shows that $A_{0}^{*} R \cap B_{0}^{*} R$ is nilpotent. Since

$$
A_{0}^{*} R \cap B_{0}^{*} R=\left(A_{0} \cap B R\right) R \cap\left(B_{0} \cap A R\right) R=A_{0} R \cap B_{0} R=A_{0} F \cap B_{0} F=F_{0},
$$

we have that $F_{0}$ is nilpotent. This contradiction completes the proof of Theorem B. 


\section{REFERENCES}

1. B. Amberg, Artinian and noetherian factorized groups, Rend. Sem. Mat. Univ. Padova 55 (1976), 105-122.

2. B. Amberg, S. Franciosi and F. de Giovanni, Groups with a nilpotent triple factorisation, Bull. Austral. Math. Soc. 37 (1988), 69-79.

3. S. Franciosi, F. de Giovanni, H. Heineken and M. L. Newell, On the Fitting length of a soluble product of nilpotent groups, Arch. Math. (Basel), 57 (1991), 313-318.

4. M. L. Newell, Supplements in abelian-by-nilpotent groups, J. London Math. Soc. (2) 11 (1975), 74-80.

5. E. Pennington, On products of finite nilpotent groups, Math. Z. 134 (1973), 81-83. 1972).

6. D. J. S. Robinson, Finiteness conditions and generalized soluble groups, (Springer Verlag $441-470$

7. D. J. S. Robinson, Splitting theorems for infinite groups, Symposia Math. 17 (1976),

8. D. J. S. Robinson, The vanishing of certain homology and cohomology groups, J. Pure Appl. Algebra 7 (1976) 145-167.

9. D. J. S. Robinson, Cohomology of locally nilpotent groups, J. Pure Appl. Algebra 48 (1987), 281-300.

10. Ya. P. Sysak, Products of infinite groups, Akad. Nauk Ukr. SSR, Preprint 82.53 (1982).

11. H. Wielandt, Subnormalität in faktorisierten endlichen Gruppen, J. Algebra 69 (1981), $305-311$.

S. Franciosi

IstrTuto di MATEMATICA

FACOLTÀ DI SCIENZE

UNIVERSITÀ di SALERNo

I-84100 SALERNo

ITALY
F. de Giovanni

Dipartimento di Matematica

UNIVERSITÀ DI NAPOLI

Via Mezzocannone 8

I-80134 NAPOLI

ITALY 